\title{
IDENTIFICATION AND CONFIDENTIALITY FOR E-GOVERNMENT
}

\author{
Reinhard Posch ${ }^{1}$, Herbert Leitold ${ }^{2}$ \\ 1 Chief Information Officer (CIO), Federal Republic of Austria, \\ Reinhard.Posch@cio.gv.at \\ 2 Secure Information Technology Center-Austria (A-SIT), \\ Herbert.Leitold@a-sit.at
}

\begin{abstract}
The omnipresence of personal computers (PCs) and the Internet has encouraged public authorities to provide citizens with means of using information and communication technologies (ICT) to contact the public services. In such e-Government environments security is a major concern. In particular the unequivocal identification of the citizen that requests services from the authorities and the confidentiality of the data that is communicated during the transactions are major concerns.

In this paper we describe how the Austrian federal government meets the security challenges that appear when advancing to e-Government. The strategic decisions and organizational structures that have been implemented to achieve coherent solutions are described. The main vehicles employed are electronic signatures and identification based on the citizen registration system. The Austrian citizen card concept that builds an underlying security infrastructure based on smart card technology is presented. The paper discusses how identification is provided with respect to data protection requirements. Regarding confidentiality we describe a model consisting of three security levels to meet the security demands of the different application domains. Moreover a concept is presented that relies upon open interfaces to achieve technology-neutrality and forward-compatibility. An example of an e-Government application is given to illustrate the flexibility of the concepts that have been followed.
\end{abstract}

Keywords: e-Government, citizen card, citizen identification, identity link, security layer

The original version of this chapter was revised: The copyright line was incorrect. This has been corrected. The Erratum to this chapter is available at DOI: 10.1007/978-0-387-35696-9_19 


\section{INTRODUCTION}

The Austrian federal government has recently carried out several major steps towards including ICT into the business processes of public authorities for both the authority's applications and for improving the business relationship between the citizens and the administrative bodies. Such support of public services by means of ICT is commonly referred to as eGovernment. Among the courses that have been set are trend setting strategic decisions, as well as statutory provisions. The major decisions that form the basis for large-scale deployment of e-Government are given below, as follows:

- The Austrian signature law [1] which is based on the EU electronic signature directive [2] and which entered into force beginning of 2000 defines that an electronic signature that fulfils certain technical requirements fulfils the requirements of writing in the same manner as a handwritten signature in a paper-based environment. The technical requirements of such electronic signatures - we refer to such electronic signatures as 'secure electronic signatures' throughout the remainder of this paper - are laid down in the signature order [3].

- In a November 2000 cabinet council an unanimous decision has been reached to use smart card technology to improve the citizen's access to public services. This led to an approach that is referred to as the 'Austrian citizen card concept' [4] where numerous smart card initiatives such as the Austrian public identity card, the Austrian health insurance card, and private sector borne smart cards such as automatic teller machine cards will fulfill the basic requirements to be used with e-Government applications. We will precise these basic requirements in section 4 .

- A number of official proceedings statutes have been amended to enable the use of electronic media in public services. Among these are provisions on how the citizen registry may be used for identification purposes, which has been defined in the administration reform law [5]. The identification process will be further discussed in section 2 . Moreover, the notification delivery law [6] has been adapted to allow for conclusion of proceedings by electronic means.

Besides the expression of the political will of advancing toward e-Government and besides the legal provisions a coordinated strategy is required to achieve lasting solutions. Given the multitude of applications carrying different characteristics an uncoordinated deployment carries the risk of isolated applications that cut itself off sweeping solutions that are based on a common infrastructure. Moreover, the federal state machinery that allocates responsibilities to the federal ministries, to the provincial 
governments, and to the municipalities, results in a number of players that gain from coordinated e-Government strategies.

In order to support such coordinated strategies a so-called ICT board has been established in 2001. The board consists of the chief information officers (CIO) of the ministries and a chairperson. The chairperson of the board is entrusted the duties of a CIO of the federal government as a staff position and reports to the cabinet through the chancellor, the vicechancellor, respectively. Cooperation with the federation of the cities and of the municipalities, as well as with the federal states is provided. In this structure, which is illustrated in figure 1 , coordinated decisions are possible that each stakeholder identifies oneself with and therefore the implementation in the various competences is provided. An operational unit has been established that implements the decisions of the board.

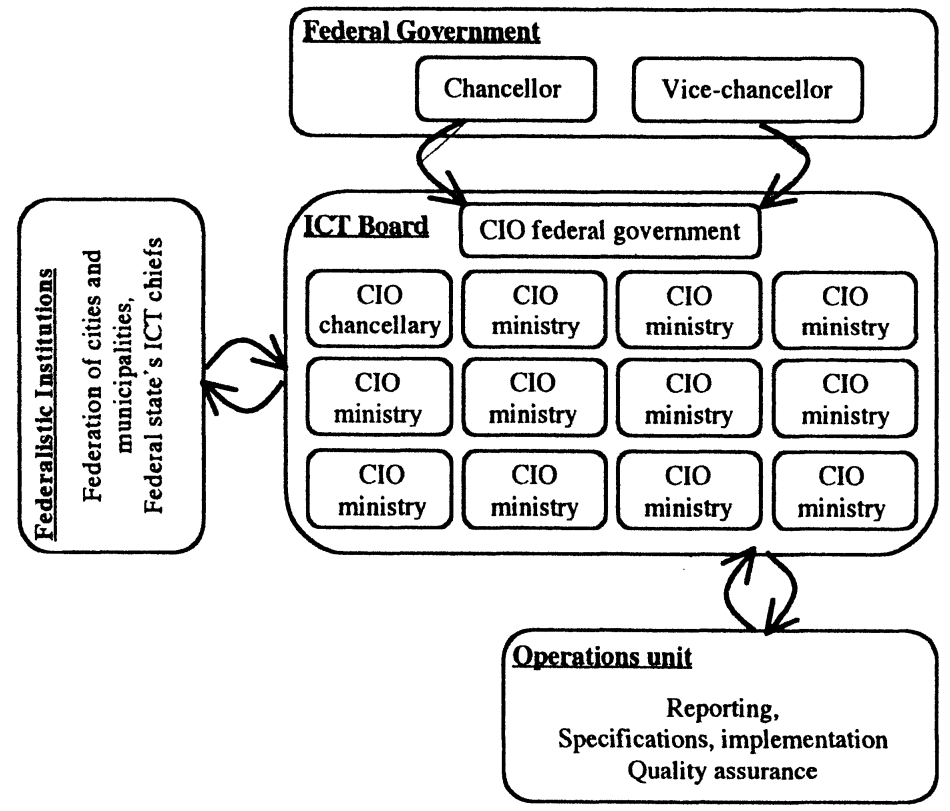

Figure 1. Organisational structure of the coordinated e-Government strategy

The ICT board that is depicted in figure 1 is a coordinating unit that translates the political provisions to the technical level by passing resolutions that - with the assistance of the operational unit - lead to specifications. Among the areas covered are some that carry specific security requirements:

- Multi-channel access: The citizen may approach the authorities by various means. While Web-based access and email will be predominant in initial phases, other access methods are conceivable. The strategic coordination needs to define general requirements in particular regarding the basic security requirements. 
- XML forms: Standardized forms based on the extensible markup language (XML) [7] are being developed. The flexibility of XML and standardized electronic signature formats for authentication [8] [9] make XML suitable in e-Government environments.

- Electronic notifications: Official proceedings need to take into consideration that citizen's life matters may change, such as the fact of possession of a PCs. Thus electronic notifications need to preserve validity even when being printed on paper. Standardized XML forms and style sheet transformations [10] assist in defining forms that can be transformed back and forth from its electronic representation to paper, whilst being capable of preserving electronic signatures appended to it.

- Electronic delivery: Secure means of delivering electronic notifications are being defined, which can provide non-reputability of receipt in the same manner as registered letters.

Besides the aspects sketched above, official proceedings are in particular concerned with unequivocal identification of the citizen, confidentiality of data communicated in the transactions, and authentication. The remainder of this paper discusses how these aspects are implemented in the Austrian e-Government initiatives: In section 2 unequivocal identification of the citizen is addressed. Section 3 continues by presenting a model that defines different security levels depending on the identification and confidentiality requirements of the applications. In section 4 the concept of a so-called security layer is introduced: By means of an open interface on a high abstraction level a solution to the problem of integrating new security technologies as they appear on the market is discussed. An example of an e-Government application following the concepts that are described in this paper is given in section 5. Finally, conclusions are drawn.

\section{IDENTIFICATION - THE PRIME GOAL}

Many official proceedings require that the citizen be unmistakably identified. Identification may for instance be needed to ensure that the person approaching the authority is the one filing the application, such as applying for a driving license, or that the person is eligible to receive certain information, such as his penal record.

In paper-based proceedings with personal appearance, the identity can be evidenced by means of identity cards, deeds, or a witness. When advancing to e-Government personal appearance certainly shall be substituted by other means. One might consider a public key infrastructure (PKI) issuing citizen's certificates suitable for identification purposes. However, although PKI certificates such as X.509 are unequivocal e.g. due to the serial 
numbers, the certificate holder is usually just indicated by the name. This does not give unmistakable identification of the person when considering equal names. Even if the certification service provider (CSP) carries out registration based on personal appearance and identity cards, unequivocal identification of the citizen than requires that the authority within the course of the official proceeding has access to both the certificate and the registration records of the CSP.

Access to the registration records of the CSP would be granted, if the CSP services are provided by governmental organizations. Such an approach has been unfavorable: On the one hand, such an approach would to some extend result in closed systems, where private sector CSPs established in the market are excluded from the e-Government marketplace. On the other hand, providing CSP services would extend the authority's scope of duties beyond its core competences.

With the introduction of a central citizen registry in Austria in 2002, a unique identifier called a central registration number (CRN) has been established that in principle allows unequivocal identification of the citizen and thus eliminates the need to access an CSP's registration record. This idea has been followed. However, data protection concerns need to be taken serious. The storage of the CRN with the proceeding's files is inhibited by law. While simply using the CRN fro identification is therefore not possible, the amended official proceedings law [5] permits to make use of a derived identifier that is specific for each type of official procedure and that does not allow to calculate the CRN. Such a procedure-specific identifier (PSI) is constructed by merging the $\mathrm{CRN}$ with an identifier of the proceeding, such as a tax declaration ID, and applying a cryptographic hash function. This is illustrated in the following figure 2 .

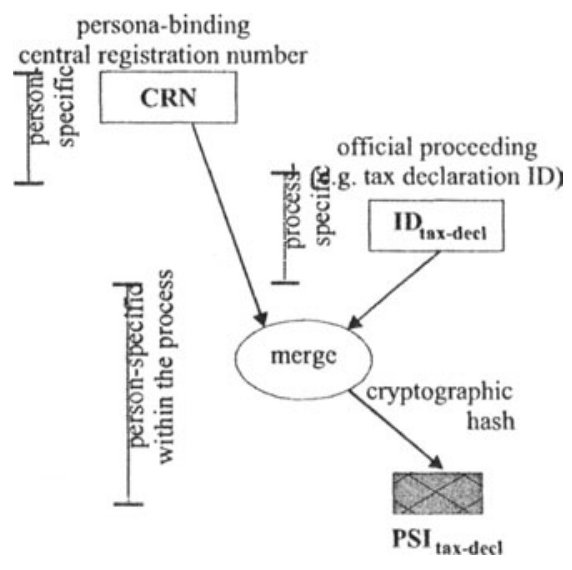

Figure 2.Construction of a procedure-specific identifier (PSI) for a tax declaration 
The CRN is part of a record that is referred to as the 'persona-binding'. The persona-binding consists of the name, the date of birth, and the CRN of the citizen and is electronically signed by the federal ministry for the interior to preserve authenticity. The persona-binding is under control of the citizen - it is stored with the Austrian citizen card that is discussed in section 4 and may be protected by the citizen by authentication codes, such as a personal identification number (PIN).

With combining the CRN with the procedure-specific ID - the tax declaration ID in the sample case illustrated in figure 2 - the citizen is uniquely identified within the official proceeding. Thus the PSI has the quality of e.g. a taxpayer's account within the tax office's application. Likewise a PSI constructed from the CRN and the process ID of an application for a preventive medical checkup has the quality of a social security number in the health insurance's application. What is gained due to the cryptographic hash is that neither the CRN can be derived from the PSI, nor can the official proceedings - in our sample cases 'tax declaration' and 'application for preventive medical checkup' - be connected due to using the same personal identifier, which would be inadmissible.

\section{CONFIDENTIALITY LEVELS}

Confidentiality is a fundamental requirement when carrying out official proceedings. As the security requirements of e-Government applications vary, it is advisable to adapt the minimum level of security required to the characteristic of the case: While providing confidentiality in anonymous information retrieval systems may by advisable to e.g. not disclose that a certain form has been downloaded, such as the application for medical checkup in our sample case, it is in this case not required to base the establishment of session keys on strong authentication and on identification of the citizen - such identification may even be inappropriate for concerns of privacy and acceptance. On the other hand, other cases may require both confidentiality and authentication. An example of such a case is to call for a penal record where that record shall not be disclosed and the citizen which the penal record refers to is the only person eligible to retrieve the record, thus requiring identification of the parties.

Three security-levels have been defined, where each level may have sublevels 'high (H)' and 'low (L)' to distinguish the strength of function. The three security levels are:

- Level I: Secure for normal use

- Level II: Secure with trustworthy infrastructure sub-levels IIL \& IIH

- Level III: Technical end-to-end security sub-levels IIL \& IIIH 
The first security level 'secure for normal use' covers the numerous use cases where confidentiality is required, but no identification of the citizen is needed. Technologically, this may rely on security measures that are readily integrated into off-the-shelf software such as secure socket layer (SSL), transport layer security (TLS) [11], respectively. More precisely, hypertext transfer protocol over SSL/TLS (HTPS) [12] for Web-based client server communication is employed. HTTPS is available with basically all Webbrowsers currently available on the market. The authority's Web-server is authenticated by means of server certificates.

The second security level 'secure with trustworthy infrastructure' employs the confidentiality provided with security level I, i.e. a HTTPS channel between the citizen's Web-browser and the authority's Web-server. In addition, identification of the citizen using the process-specific identifier PSI is needed, as discussed in the previous section 3 (cf. figure 2). Authentication is provided by an electronic signature. The computation of the PSI out of the CRN kept with the persona-binding stored on the citizen card and the identifier of the official case stored at the server side requires processing capabilities. An active component is therefore required that either runs on the authorities Web-server, i.e. the citizen's CRN is communicated to the active component at the server, or the active component runs at the citizen's side. These two cases distinguish the strength of function IIL for the additional active component at the server side (e.g. a JAVA servlet) or IIH for an active component installed with the citizen's PC. The case IIL is further discussed as an example in section 5 .

The trustworthy infrastructure indicated in security level II refers to relying on the security measures provided by the off-the-shelf application the HTTPS implementation of the browser, and combining this with authentication by means of an electronic signature and identification with the PSI, both provided by the citizen card. In a few cases, placing reliance on the software component (the browser) may not be sufficient. In the third security level 'technical end to end security' the establishment of session certificates for mutual authentication and session confidentiality is delegated to the hardware component - the citizen card - as well. The browser component is just entrusted the content encryption based on session keys established by the citizen card. A distinction into sub-levels IIIH and IIIL is made on whether the authentication is carried out by a secure electronic signature that requires assured technical quality or whether a second key pair is used.

With the identification model and the provision of confidentiality, two of the three fundamental security requirements in e-Government have been addressed. The third requirement - provision of secure electronic signatures 
- is discussed in the following section, together with the important aspect of remaining technology-neutral to allow for inclusion of future technologies.

\section{TECHNOLOGY-NEUTRAL SECURITY INTERFACE}

Official proceedings are characterized by requirements of writing and signatures. As the Austrian signature law [1] lays down that secure electronic signatures fulfill the requirements of handwritten signatures, the legal basis for such processes that require the written form is given ${ }^{93}$. The technical requirements for secure electronic signatures are basically, that they are based on so-called qualified certificates where the CSP issuing the qualified certificate has to meet certain requirements, and that the secure electronic signature needs to be created by a so-called secure signaturecreation device (SSCD), which is the device that implements the signaturecreation data - the private key. In addition, the security-relevant elements such as the PIN entry to create a signature need to meet certain criteria. The devices involved in the process, such as the SSCD or the trustworthy systems used by the CSP, are assessed by notified bodies.

Details of the technical requirements are given in the signature order [3], such as the signature suites eligible. For instance Rivest, Shamir, Adleman (RSA) [13] and digital signature algorithm (DSA) [14] with 1023 bit keys are considered secure until end of 2005, DSA variants based on elliptic curves [15] [16] with 160 bit keys, respectively.

Obvious candidates to fulfill the requirements for SSCDs that readily are capable of implementing the algorithms required and capable of giving the required physical protection of the private keys are smart cards. However, technology evolves rapidly and other solutions for SSCDs may show up in the market, such as personal digital assistants or cell phones. The parameters of the algorithms such as the key sizes may change. The interface to the smart card such as the commonly used cryptographic token standard [17] may be subject to revisions, or the storage capacity of smart cards certainly will increase over time. To adapt all the e-Government applications whenever such technology changes occur, such as integrating the modules for larges key sizes, certainly will turn out a costly experience. A

93 A few exceptions are laid down in the Austrian signature law [1] where secure electronic signatures do not have the effect of written form. Examples are declarations that require a notarial deed or transactions under inheritance law. We do not specifically address these cases in this paper and for concerns of simplicity we therefore assume the legal equivalence between a handwritten signature and a secure electronic signature. 
technology-neutral approach is advisable that avoids such problems when integrating new technologies.

Actually, the e-Government application anyhow does not need to be aware of technology changes. From a process perspective, to assess whether the requirement of written form is met by electronic means, the application needs confidence that the secure electronic signature has been verified, regardless whether the SSCD is a smart card, or regardless whether RSA, DSA, or elliptic curve cryptography has been employed. Both the creation of a secure electronic signature at the citizen's PC and the verification of the secure electronic signature at the server side can be delegated to a module that is accessed via an interface on a high abstraction level. We refer to such a module containing the security-relevant functions of signature creation and signature verification as a security capsule. The interface to access the security capsule is called the security layer.

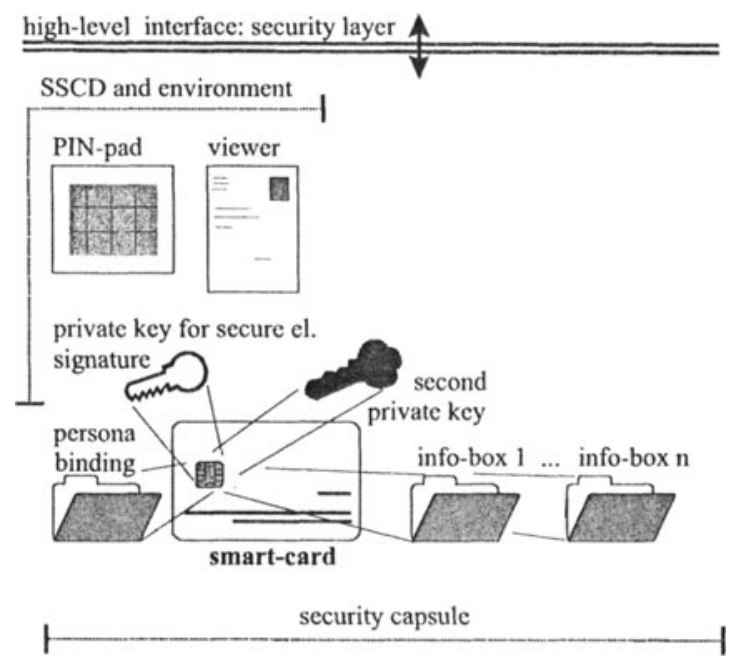

Figure 3. The security layer as a technology-neutral interface

An example of a security-capsule is illustrated in figure 3. The SSCD consists of the smart card implementing the private key for the secure electronic signature, its environment consists of the PIN-pad to create a signature and a trusted viewer component to display the data to be signed. Creation of a secure electronic signature can than be triggered on a high abstraction level be communicating the document to be signed via a highlevel interface - the security layer - and requesting signature-creation.

In addition to signature creation components, figure 3 shows a second private key which e.g. can be used for 'general electronic signatures' that are not assumed to substitute a handwritten signature, or for establishing session 
certificates for a TLS connection. Moreover, storage containers that may hold the persona-binding for identification purposes with the PSI scheme discussed in section 2 are shown, as well as further data storage denoted 'info-boxes' that may hold mandates, certificates, or other data. All these functions, such as establishing session keys for content encryption, or reading or writing info boxes may be done on a high abstraction level via the security layer.

Actually, figure 3 shows the minimum requirements that have been defined as the Austrian citizen card concept. A smart card (or any other technical solution) is considered an Austrian citizen card, if the following requirements are fulfilled:

- The citizen card needs to be capable of creating secure electronic signatures, i.e. the citizen card needs to be a SSCD,

- A second private key for authentication or confidentiality needs to be implemented,

- Info-boxes to store the persona-binding (including the citizen's CRN), or for certificates, mandates or other data need to be provided,

- All these functional blocks need to be accessible via a single interface, the so-called security layer.

The security layer is specified as a request/response scheme coded in XML. Examples of such XML requests are the request of creating a secure electronic signature following the cryptographic message syntax (CMS) [18] or XMLDsig syntax [8], the verification of a CMS or XMLDsig signature, or access to an info-box. The corresponding responses are the signed data, an indication of the signature verification result, or an indication of the info-box $\mathrm{r} / \mathrm{w}$ request, error codes in case of a failure, respectively. A number of socalled transport bindings have been specified, i.e. the protocols to access the security capsule. These are mainly based on transmission control protocol, internet protocol (TCP/IP). The transport bindings include access via simple TCP sockets for the transmission of the XML requests and responses, HTTP, or HTTPS.

With that scheme, technology-neutrality and forward-compatibility are provided in a manner that the changes required when major technology progresses need to be considered are limited to a single entity - the security capsule. This shall enable easy inclusion of upcoming technologies by keeping the costly back-office applications unchanged. In the following section we give an example on how authentication and identification is implemented in that concept. 


\section{E-GOVERNMENT EXAMPLE}

In this section, we give a case study on how the concepts that have been introduced in this paper can be employed to implement e-Government. We take requesting penal record as our sample case. Actually, this a quite frequent process, as e.g. fresh penal records are required for tenders when placing public bids. The sample case 'penal record' has not just been chosen for its frequent occurrence. When neglecting payment of the administrative fee, which we do for concerns of simplicity, it is a quite simple process: Once the citizen is uniquely identified by the server, the citizen's penal record can be delivered. In addition, confidentiality of the data transmitted is required. Thus, with reference to the confidentiality levels discussed in section 3 , we assume the security requirement of type 'level IIL'. I.e. identification is required and the active component for creating the processspecific identifier PSI may run at the server side.

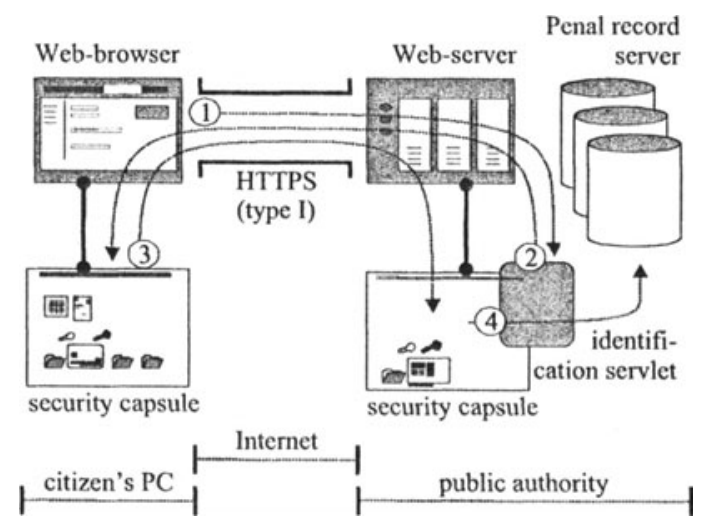

Figure 4. e-Government example

Figure 4 illustrates the process. Initially, at both sides the citizen's PC and the authority's Web-server a security capsule is running. In addition, the authority's Web-server has an active component installed for controlling the identification and authentication process. In our sample case, the active component is a JAVA servlet. To initiate the delivery of a penal record, four steps - indicated as circles in figure 4 - are required, as follows:

1. The citizen accesses the Web-server of the public authority. To initiate the process the citizen connects to the active component (the identification servlet) using a HTTPS connection, such as via a link at the Web-server. Thus, with reference to section 3 , the confidentiality level is of type I at that stage. 
2. The identification servlet authenticates itself by creating a so-called secure communication token (SCT). The SCT consists of the unique resource identifier (URI) of the identification servlet and time mark. The SCT is signed by the security capsule at the public authority's site and is transferred via the TLS connection.

3. The citizen's Web-browser accesses the citizen's security capsule. This is done under the Web-server's control by either javascript or by HTTP redirects. The SCT's signature is verified and the citizen's personabinding is appended to the SCT. The resulting structure, we refer to it as the identity link, is signed with the citizen's security capsule and transferred to the identification servlet. Note, that both releasing the persona-binding and creating the electronic signature may require entering authorization codes, such as the citizen entering a PIN. This is done under control of the security capsule and transparent to the application.

4. Finally, the identification servlet verifies the citizen's signature appended to the identity link. The signature verification process includes retrieval of certificate status information such as a certificate revocation list (CRL). The identification servlet extracts the CRN from the persona-binding and constructs the process-specific identifier PSI. This data is forwarded to the back-office application - the penal record server in our sample case.

Although we neglected the administrative fees in the beginning of this section for concerns of simplicity, its inclusion does not complicate the procedure too much. The payment of the administrative fees may be enclosed as a XML confirmation of payment signed by a bank. Such signed confirmations of payment are currently being implemented.

\section{CONCLUSIONS}

The paper has discussed the e-Government approaches that are followed by the Austrian government. The legal provisions and strategic decisions that have been made to enable electronic means in the business relationship between the public authorities and the citizen have been presented. These are mainly the signature law and official proceedings statutes that have been amended, as well as the trend-setting decision to employ smart cards as means of easing the citizen's access to public services. The structures to enable coordinated proceeding have been presented.

Regarding technical aspects, the paper has presented the method of deriving an identifier from the citizen's central registration number in a way that the citizen is uniquely identified within that certain official proceeding, but which maintains data protection requirements. In addition, a model of 
three confidentiality levels has been presented that fits the security requirements of the various e-Government applications.

The Austrian citizen card concept has been discussed as a model that provides technology-neutrality by means of an open interface called the security layer. This interface hides the internals of the citizen card implementation from the e-Government application. Thus, technological progresses can easily be integrated by replacing a single entity - called the security-capsule. The flexibility of the concept has been demonstrated on a simple e-Government case study - a request for a penal record.

\section{REFERENCES}

[1] Austrian signature law: "Bundesgesetz über elektronische Signaturen (Signaturgesetz SigG)", BGBl. I Nr. 190/1999, BGBl. I Nr. 137/2000, BGB1. I Nr. 32/2001.

[2] Directive 1999/93/EC of the European Parliament and of the Council of 13. December 1999 on a community framework for electronic signatures.

[3] Austrian signature order: "Verordnung des Bundeskanzlers über elektronische Signaturen (Signaturverordnung - SigV)", StF: BGBI. II Nr. 30/2000.

[4] Posch R., Leitold H.: "Weissbuch Bürgerkarte", Bundesministerium für öffentliche Leistung und Sport, IT-Koordination des Bundes, June 2001.

[5] Administration reform law: "Verwaltungsreform Gesetz", 2001 amending the general offical proceedings law "Allgemeines Verwaltungsverfahrensgesetz (AVG)" BGBl. Nr. $51 / 1991$.

[6] Notification delivery law: "Bundesgesetz vom 1. April 1982 über die Zustellung behördlicher Schriftstücke”, BGBI. I Nr. 137/2001.

[7] Murata M, Laurent S. St., and Kohn D.: "XML Media Types", IETF Request For Comment RFC 3023, 2001.

[8] Eastlake D., Reagle J., and Solo D.: "XML-Signature Syntax and Processing", W3C Recommendation, 2002.

[9] ETSI SEC: "XML Advanced Electronic Signatures (XAdES)", Technical Specification ETSI TS 101903, 2002.

[10] Clark J.: "XSL Transformations (XSLT)", W3C Recommendation, 1999.

[11] Dierks T., Allen C.: “The Transport Layer Security (TLS) Protocol, Version 1.0”, IETF Request For Comment RFC 2246, 1999.

[12] Rescorla: "HTTP over TLS”, IETF Request For Comment RFC 2818, 2000.

[13] RSA Laboratories: "RSA Cryptography Standard", PKCS \#1 v2.1 draft 2, 2001.

[14] National Institute of Standards and Technology, "Digital Signature Standard (DSS)", NIST FIPS Publication 186-2, 2000.

[15] American National Standards Institute, "Public Key Cryptography for the Financial Services Industry: The Elliptic Curve Digital Signature Algorithm (ECDSA)", ANSI X9.62-1998, 1998.

[16] International Organization for Standardization, "Information technology - Security techniques - Cryptographic techniques based on elliptic curves - Part 2: Digital signatures", ISO/IEC FCD 15946-2, 1999.

[17] RSA Laboratories: “Cryptographic Token Interface”, PKCS \#11 v2.11, 2001.

[18] Hously, R.: “Cryptographic Message Syntax (CMS)", IETF Request for Comment RFC 2630, 1999. 